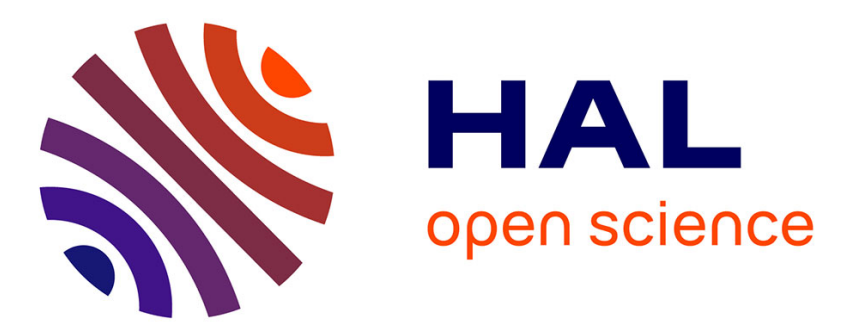

\title{
A multi-channel Sigma Delta modulator for subband digital predistortion with LTE signals
}

\author{
Kelly Tchambake, Germain Dang-Kièn Pham, Chadi Jabbour, Patricia
}

Desgreys, Patrick Loumeau

\section{- To cite this version:}

Kelly Tchambake, Germain Dang-Kièn Pham, Chadi Jabbour, Patricia Desgreys, Patrick Loumeau. A multi-channel Sigma Delta modulator for subband digital predistortion with LTE signals. IEEE International New Circuits and Systems Conference (NEWCAS), Jun 2016, Vancouver, Canada. pp.1 - 4, 10.1109/NEWCAS.2016.7604802 . hal-01613851

\section{HAL Id: hal-01613851 \\ https://hal.science/hal-01613851}

Submitted on 10 Oct 2017

HAL is a multi-disciplinary open access archive for the deposit and dissemination of scientific research documents, whether they are published or not. The documents may come from teaching and research institutions in France or abroad, or from public or private research centers.
L'archive ouverte pluridisciplinaire HAL, est destinée au dépôt et à la diffusion de documents scientifiques de niveau recherche, publiés ou non, émanant des établissements d'enseignement et de recherche français ou étrangers, des laboratoires publics ou privés. 


\title{
A Multi-Channel $\Sigma \Delta$ Modulator for Subband Digital Predistortion with LTE signals
}

\author{
Kelly Tchambake, Dang-Kièn Germain Pham, Chadi Jabbour, Patricia Desgreys, Patrick Loumeau \\ Télécom ParisTech, CNRS/LTCI UMR 5141, Université Paris-Saclay \\ Email: kelly.tchambake@telecom-paristech.fr
}

\begin{abstract}
With the increasing bandwidth of communication signals, the challenges on the feedback path used for digital predistortion (DPD) of power amplifiers are getting significantly stringent. A subband approach has been recently proposed by the authors in order to relax the design constraints on the feedback path ADC. This paper presents a new step toward a practical implementation of the concept with LTE signals. A continuous-time multi-channel modulator is proposed to address wider bandwidths and sizing choices are discussed with simulation results. Some nonlinear effects of the feedback path such as I/Q imbalance, pure nonlinearity and quantization are simulated to define high level design parameters. Results show that for a distorted signal with $30 \mathrm{~dB}$ ACPR, 60 dB SNR is sufficient for the principal subband and 22 dB SNR is required for the adjacent subbands to perform as good as an ideal fullband DPD. Continuous-time simulation using a 2-4-4 configuration of the zero IF MSNBC with 4-bit quantizers achieves the required subband SNRs.
\end{abstract}

\section{INTRODUCTION}

Due to stringent linearity constraints in order to maximize mobile networks capacity, communication systems use power hungry power amplifiers (PA) to meet standard requirements. An attractive approach to break the linearity-efficiency tradeoff is digital predistortion (DPD) [1]. This technique requires a feedback path to digitize the distorted signal at the output of the PA. Because of PAs nonlinearity, output signal undergoes a spectral regrowth resulting in very wideband signal that puts stringent design constraints on the feedback path blocks. More precisely, if the useful signal bandwidth is $B W$, after distortion it becomes $M \times B W$ with $M$ the highest significant nonlinear order.

Forthcoming communication standards like LTE-A specify transmission scenarios using signals with up to $100 \mathrm{MHz}$ bandwidth Conventional DPD requires to digitize the distorted output signal at least up to the $5^{\text {th }}$ order of nonlinearity. In the case of a $100 \mathrm{MHz}$ LTE-A signal, this results in a digitization bandwidth of $500 \mathrm{MHz}$. In order to relax design constraints of the feedback path, some DPD approaches focus on limiting the feedback bandwidth [2] [3] at the cost of significant processing resources. An alternative approach presented in [4] digitizes the signal in subband which relaxes the analog-to-digital converter (ADC) design and enables the digital processing to operate at lower frequencies.

The power level of the spectral regrowth is usually $40 \mathrm{~dB}$ to $60 \mathrm{~dB}$ lower than the power of the desired signal. A fullband single ADC will require a high dynamic range (DR) in $M \times B W$ bandwidth in order to capture the carrier and low power distortion signals. With the subband approach, the main signal band and adjacent bands can have different DR requirements. Sigma Delta $(\Sigma \Delta)$ modulators can be advantageously used for this subband approach thanks to their ability to digitize bandpass signals without additional mixers and costly filters while minimizing power consumption. The multistage noise band cancellation (MSNBC) ADC has been proposed to optimize the ADC structure to the DR requirements [4]. A discretetime implementation of this ADC for WCDMA signals was presented in [5]. However, for wideband applications, continuous-time (CT) modulators are preferred to their discrete-time counterpart due to their better power efficiency and inherent anti-aliasing filter [6]. With CT implementation, new practical constraints that were not considered in the discrete-time implementation of the MSNBC arise.

This paper is organized as follows: section II presents the CT MSNBC architecture. Section III focuses on the impact of multiband ADC noise and nonlinearity on DPD performance. Section IV sets ADC design constraints and section $\mathrm{V}$ concludes this paper.

\section{THE MSNBC ARCHITECTURE}

The MSNBC modulator has the feature to implement several DR specifications depending on the number of channels to digitize. An illustration of its mode of operation is presented in Fig. 1.

\section{A. Subband operation}

The MSNBC consists of several $\Sigma \Delta$ modulators. The primary modulator $\Sigma \Delta_{0}$ is centered at $F_{c 0}$ and digitizes the high power subband of bandwidth $B W$. Modulators $\Sigma \Delta_{1 A}$ and $\Sigma \Delta_{1 B}$, respectively centered at $F_{c 0}+B W$ and $F_{c 0}-B W$ digitize the first adjacent subbands of bandwidth $B W$. Second adjacent subbands are digitized by $\Sigma \Delta_{2 A}$ and $\Sigma \Delta_{2 B}$ modulators centered at $F_{c 0} \pm 2 B W$.

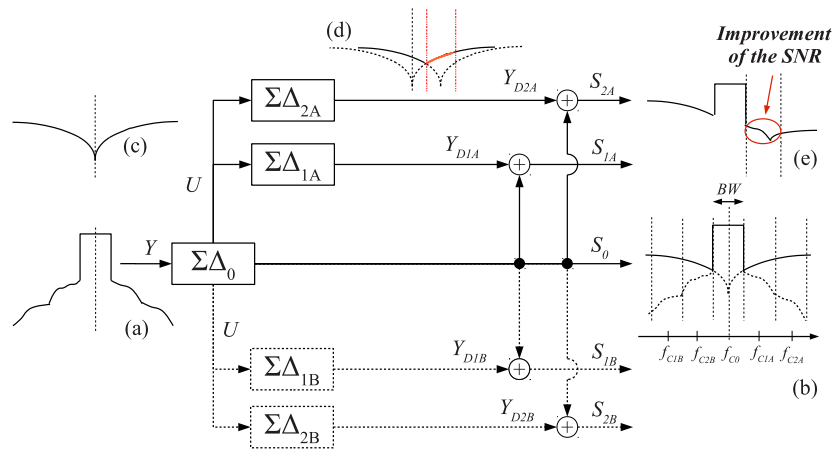

Fig. 1. MSNBC modulator operating mode

The distorted signal $Y$ illustrated by (a) in Fig. 1 is applied to the ADC input. The discrete-time linear model of the output signal of $\Sigma \Delta_{0}$, is:

$$
S_{0}(z)=S T F_{0}(z) Y(z)+N T F_{0}(z) N_{0}(z),
$$

with $S T F_{0}$ and $N T F_{0}$ respectively the signal and noise transfer functions of $\Sigma \Delta_{0}$, and $N_{0}$ its quantization noise. This signal is shown in Fig. 1 (b). Due to quantization noise, $S_{0}$ is not adapted to accurately predistort the input signal of the PA and the adjacent channels should be digitized for an accurate DPD. Assuming for the 
sake of simplicity a unitary STF in the band of operation, the signal $U$ defined by $U=Y-S_{0}$ can be seen as a negative version of $\Sigma \Delta_{0}$ shaped quantization noise. We introduce for this architecture the residual signal transfer function (RSTF) defined as

$$
R S T F=1-S T F .
$$

This function represents the cancellation of $Y$ in $U$. Fig. 1 (d) illustrates $Y_{D 1 A}$, the output of $\Sigma \Delta_{1 A}$ made of $U$ and $\Sigma \Delta_{1 A}$ shaped quantization noise. Assuming again a unitary STF in $\Sigma \Delta_{1 A}$, the quantization noise of $\Sigma \Delta_{0}$ is canceled in the first adjacent subband when $S_{0}$ and $Y_{D 1 A}$ are added, as represented in Fig. 1 (e). This leads to an improvement of the signal-to-noise ratio (SNR) in this subband. The same principle applies to other adjacent modulators and enables to improve the SNR.

\section{B. IF vs. zero IF architecture}

As shown in Fig. 1, the MSNBC modulator consists of several $\Sigma \Delta$ modulators with different center frequencies: $F_{c 0}, F_{c 0} \pm B W$ and $F_{c 0} \pm 2 B W$. Thus, two implementations of this modulator are possible: at an intermediate frequency $(I F)$ with $F_{c 0}=F_{S} / 4$ for example, where $F_{S}$ is the sampling frequency of the modulator, or at zero $I F$ with $F_{c 0}=0 \mathrm{~Hz}$.

After studying and comparing the two architectures, it was decided to adopt the zero IF architecture for several considerations. First, in a zero IF architecture, the NTF complex conjugate zeros are closer to each other, which improves significantly the SNR. Besides, the zero IF architecture requires to design less different modulators compared to the IF architecture which needs as many different modulators as subbands to digitize. As presented in Fig. 2, each identical (I/Q) path of a zero IF MSNBC modulator is made of a lowpass modulator and two bandpass modulators.

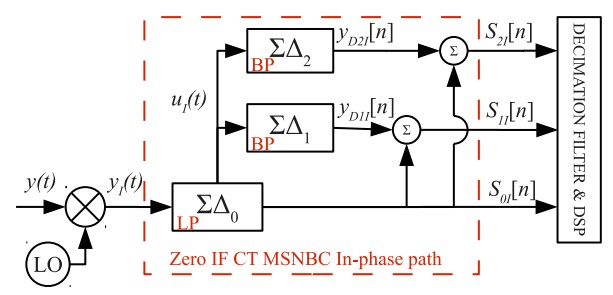

Fig. 2. In-phase path of the CT zero IF MSNBC architecture

Another important aspect in MSNBC modulators is the delay. The delay of the loop filter adds a phase shift in the RSTF and thus limits the cancelation of the input signal at node $U$. Moreover, since a CT modulator implementation has been chosen for the modulators, the architecture is sensitive to Excess loop delay (ELD). This delay has been widely studied over the past and several methods have been proposed to deal with this non ideality. In [7] some compensation methods are compared. While those techniques will maintain the STF and SNR performance of a modulator, they may degrade the RSTF. Fig. 3 compares the RSTF of a $4^{\text {th }}$ order lowpass modulator to the RSTF of a $4^{\text {th }}$ order bandpass modulator centered at $F s / 4$. For each implementation, two cases are presented: the ideal modulator without ELD and the modulator with ELD and the classical compensation [7]. It can be seen that ELD compensation considerably impacts the RSTF of the bandpass modulator. The signal attenuation around DC is close to $44 \mathrm{~dB}$ for the ideal and the ELD compensated lowpass modulator.
However, for the bandpass modulator, the RSTF notch located at $F s / 4$ is shifted because of the delay. The attenuation around $F s / 4$ drops from 36 to $5 \mathrm{~dB}$. Thus, a better RSTF is achieved with the zero IF CT MSNBC compared to the IF CT MSNBC.

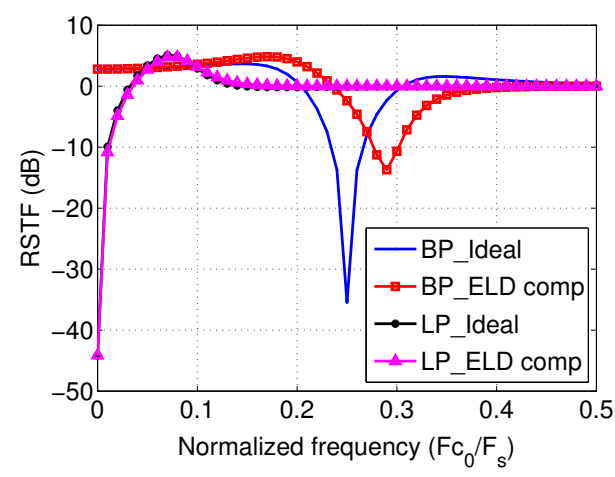

Fig. 3. ELD effect on RSTF

Finally, at circuit level, the design specifications of modulator building blocks are relaxed for the zero IF architecture as the signal frequency band is low.

Nonetheless, the performance of the zero IF architecture could be affected by specific non idealities especially I/Q mismatch. Its impact on the DPD performance as well as the impact of the main non idealities in the DPD feedback path will be studied in the next section in order to set adequately the ADC specifications.

\section{IMPACT ON DPD PERFORMANCE}

In this section, different nonlinear effects are successively taken into account in the DPD feedback path and their effect on DPD correction are evaluated. The focus is on I/Q mismatch, nonlinear distortion and quantization with a MSNBC ADC. Similarly to other cascaded architectures and parallel architectures, other non idealities may deteriorate the ADC performance such as mismatch of the transfer characteristics of the modulators for the digital noise cancellation. The impact of those non idealities is still under investigation. However, it is expected that they deteriorate the SNR performance without generating significant nonlinearity. Simulation results below show a better robustness of DPD performance against SNR reduction than against nonlinearity.

The correction performance are simulated using a $20 \mathrm{MHz}$ monocarrier LTE signal. This signal is distorted using the memory polynomial PA model proposed in [8]. The linearization of this system is achieved with a memory polynomial model identified by a leastsquare method [8]. The nonlinear order of the inverse model is set to 9 and its memory depth is 3 in order to provide $55 \mathrm{~dB}$ adjacent channel power ratio (ACPR) and $0.2 \%$ error vector magnitude (EVM) when all blocks in the feedback path are ideally linear and there is no quantization error.

\section{A. Mixer $I / Q$ imbalance}

I/Q imbalance is assumed to be caused by the mixer and the baseband I/Q paths. We assume in this subsection that the mixer I/Q imbalance predominates over the baseband I/Q imbalance and there 
is no quantization error. As explained in [9], the I/Q imbalance can be modeled on the complex baseband signal with the following equation:

$$
\tilde{y}(t)=\frac{1+g_{m} \mathrm{e}^{-j \Phi_{m}}}{2} y_{a t t}(t)+\frac{1-g_{m} \mathrm{e}^{j \Phi_{m}}}{2} y_{a t t}^{*}(t),
$$

where $\tilde{y}(t)$ and $y_{a t t}(t)$ are respectively the I/Q imbalanced and the ideal baseband complex envelope, $g_{m}$ is the relative amplitude mismatch between I and Q branches, $\Phi_{m}$ is the phase mismatch and $(\cdot)^{*}$ denotes the complex conjugate.

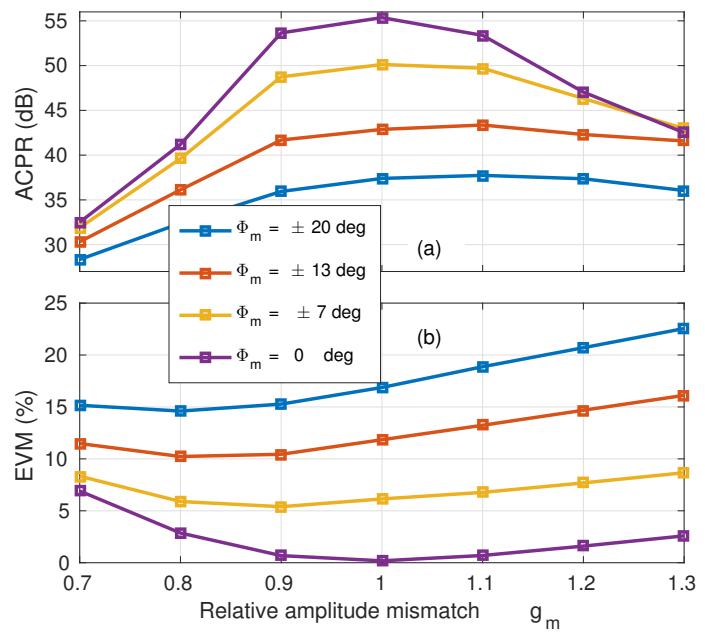

Fig. 4. DPD performance in terms of ACPR (a) and EVM (b) vs I/Q mismatch: simulation results;

The upper and lower parts of Fig. 4 show respectively the ACPR and the EVM versus the relative amplitude mismatch $g_{m}$. In both graph, each line corresponds to a given phase error $\Phi_{m}\left( \pm 20^{\circ}, \pm 13^{\circ}\right.$, $\left.\cdots, 0^{\circ}\right)$. As expected, the worst results are achieved for large values of phase error and large relative amplitude mismatch. It can be seen on Fig. 4 (a) that the relative amplitude mismatch must be such that $0.9 \leq g_{m} \leq 1.1$ and the phase error should be less than about $10^{\circ}$ to meet the 3GPP LTE standard requirements [10]. Regarding the EVM (Fig. $4(\mathrm{~b})$ ), the standard requirements are met for the same range of $g_{m}$ and $\Phi_{m}$ as ACPR.

\section{B. Fullband nonlinear feedback path}

The effect of a nonlinear distortion generated in the feedback path is now considered. This distortion may be caused by compression in the active blocks of the feedback path. We assume that distortions are modeled by a $3^{\text {rd }}$ order nonlinearity and that higher orders nonlinearities have minor effects. As mentioned in [9], this nonlinearity can be modeled on the complex baseband signal by:

$$
\tilde{y}_{B B}(t)=y_{a t t}(t)+\alpha\left(\left[y_{a t t}^{*}(t)\right]^{2}+3 y_{a t t}^{2}(t)\right) y_{a t t}^{*}(t),
$$

where $\alpha$ is the nonlinearity coefficient. We define the fullband signal to distortion ratio (SDR) as:

$$
S D R=\frac{P_{\text {mean }}\left\{y_{a t t}(t)\right\}}{P_{\text {mean }}\left\{\alpha\left(\left[y_{\text {att }}^{*}(t)\right]^{2}+3 y_{\text {att }}^{2}(t)\right) y_{\text {att }}^{*}(t)\right\}},
$$

Fig. 5 shows the ACPR and EVM of the linearized PA output obtained by simulation. As long as the distortions generated by the feedback path are low enough $(S D R \geq 55 \mathrm{~dB})$, correction performance are maximum. For $S D R \leq 55 \mathrm{~dB}$, the ACPR drops. The EVM is less sensitive to this $3^{\text {rd }}$ order nonlinearity as it remains

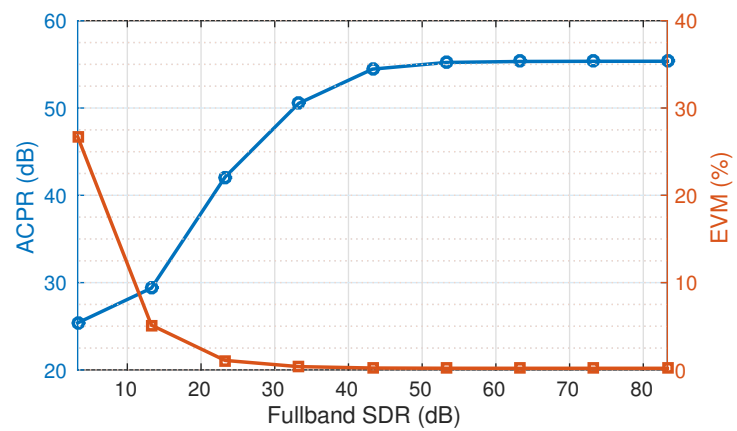

Fig. 5. DPD performance in terms of ACPR and EVM vs nonlinearity of the feedback path; (SDR stands for signal to distortion ratio)

constant for a wider range of distortion level: its effect is significant for $S D R \leq 40 \mathrm{~dB}$.

In order to cope with circuit non idealities, the target linearity of the ADC will be set to $60 \mathrm{~dB}$. These minimum linearity constraints are currently achievable with minimum careful design.

\section{Subband $A D C$ requirements}

We now consider that the distorted output of the PA is digitized by an MSNBC ADC. The MSNBC architecture provides new degrees of freedom such as the possibility of having different quantization noise level for each subband. This can be achieved by setting different quantization step sizes for each modulator quantizer. The effect of this subband quantization on the linearization results is simulated and ACPR and EVM are shown in Fig. 6. For this paper, secondary modulators use the same quantizers. The $\mathrm{x}$-axis is the SNR in the principal subband, which corresponds to the ideal $20 \mathrm{MHz}$ transmit band and each colored line represents a specific SNR in the $20 \mathrm{MHz}$ adjacent subband.
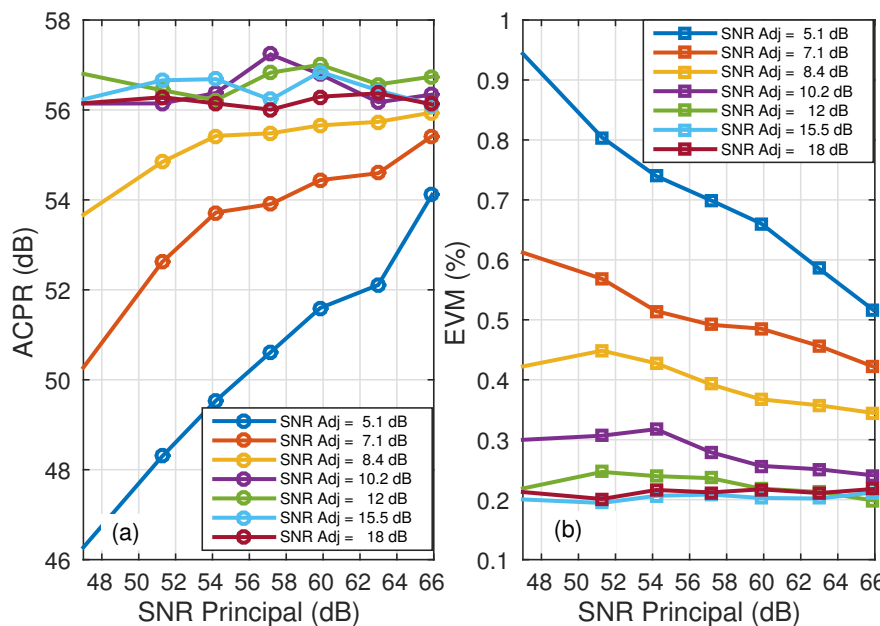

Fig. 6. DPD performance in terms of ACPR and EVM vs. subband quantization SNR; ACPR without DPD: $30 \mathrm{~dB}$; EVM without DPD: $4.3 \%$

As expected, the higher the SNR, the better the ACPR and EVM. The ACPR is independent of principal subband SNRs between 48 and $66 \mathrm{~dB}$ for adjacent subband SNR greater than $10.2 \mathrm{~dB}$. The EVM has a similar characteristic for adjacent subband SNRs greater than $12 \mathrm{~dB}$. 


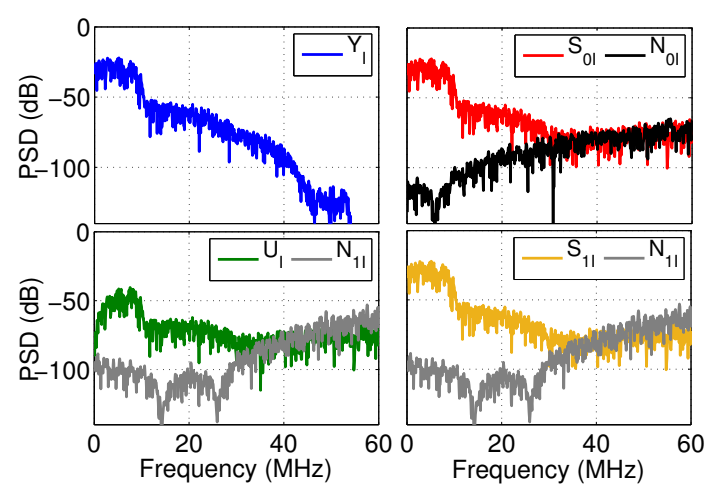

Fig. 7. MSNBC signals spectra

By considering the input signal peak-to-average power ratio (PAPR) in these simulation results, the minimum performance of the MSNBC ADC is set to $60 \mathrm{~dB}$ SNR in the principal subband and $22 \mathrm{~dB}$ SNR in the adjacent subband.

\section{TOP LEVEL DESIGN}

Section III demonstrated that $60 \mathrm{~dB}$ SNR in the principal subband and $22 \mathrm{~dB}$ SNR in the adjacent subband are required in order to obtain the same linearization performance as in the case of an ideal fullband feedback path. A $20 \mathrm{~dB}$ margin between the ADC overall noise floor and distortions and the quantization noise is chosen as a good tradeoff between power consumption and design complexity. Therefore the targeted SQNR are $80 \mathrm{~dB}$ SNR in the principal subband and $42 \mathrm{~dB}$ SNR in the adjacent subband.

Simulations are performed with a VerilogA model of the zero IF architecture presented in Fig. 2. All $\Sigma \Delta$ modulators have the continuous time cascade of integrators in feedback form (CIFB) [11] in order to avoid STF peaking. The CIFB modulator used consists of ideal continuous time integrators, clocked quantizer and scaling coefficients to realize a loop filter. Those coefficients are calculated with the Delta Sigma Toolbox [11] such that all modulators have $0 \mathrm{~dB}$ gain in their bands of operation. After exploring the design space provided by the different degrees of freedom of the architecture, i.e., order, OSR and quantizer number of bits, the configuration that gave us the best compromise is a 4-bit $2^{\text {nd }}$ order loop filter with an OSR of 32 for the primary modulator and a 4-bit $4^{\text {th }}$ order loop filter with an OSR of 16 for the secondary modulator. This results in a common sampling frequency of $640 \mathrm{MHz}$ for all the modulators. The 4-bit quantizer of the primary modulators reduces the quantization noise power sent to the secondary modulators and avoids peaking in the STF. The feedback path of each $\Sigma \Delta$ modulator is made of 4-bit non return to zero (NRZ) digital-to-analog converters (DAC) to reduce jitter contributions.

Fig. 7 presents the power spectrum density (PSD) of signals in the zero IF CT MSNBC architecture. The input signal $Y_{I}$ represents the downconverted PA output of a $20 \mathrm{MHz}$ mono carrier LTE signal. For sake of clarity, the shaped quantization noise is also shown in the spectrum. The power of the first adjacent subband nonlinearity is set $30 \mathrm{~dB}$ lower than the carrier power. As expected, $S_{0 I}$ is made of $Y_{I}$ and $\Sigma \Delta_{0}$ shaped quatization noise $N_{0 I}$. The SQNR in the main channel of $S_{0 I}$ is $84.6 \mathrm{~dB}$. $\Sigma \Delta_{0}$ quantization noise is low compared to nonlinearities in the first adjacent channel of $S_{0 I}$ leading to $30.8 \mathrm{~dB}$ SQNR. The subtraction of $S_{0 I}$ to $Y_{I}$ enables to attenuate the high power signal by $38.2 \mathrm{~dB}$ in $U_{I}$. Given the lowpass modulator RSTF presented in Fig. 3(b), the higher the signal frequency, the lower the attenuation in the main channel. $U_{I}$ is then digitized by $\Sigma \Delta_{1}$ in the adjacent channel. The sum of $\Sigma \Delta_{1}$ output signal and $S_{0 I}$ cancels $\Sigma \Delta_{0}$ quantization noise in the adjacent band and improves the SQNR by almost $15 \mathrm{~dB}$. The SQNR in the first adjacent channel of $S_{1 I}$ is then $45.9 \mathrm{~dB}$.

\section{CONCLUSION}

The MSNBC modulator is a good candidate to digitize distorted signal in subband DPD. Instead of having the same DR constraints in a wide bandwidth, this converter relaxes DR per band where the use of parallel $\Sigma \Delta$ modulators avoids to use additional mixers and filters. The zero IF implementation is better than the low IF one in terms of delay sensitivity and design constraints. Results showed that the DPD can perform proper correction when the I/Q mismatch has a phase error $\left|\Phi_{m}\right|<7 \mathrm{deg}$ and a relative gain mismatch $0.9<g_{m}<1.1$. In addition, by including margins, $60 \mathrm{~dB}$ and $22 \mathrm{~dB}$ SNR are respectively needed in the main and adjacent subbands to perform DPD as good as with ideal digitization. This can be obtained with a zero IF CT MSNBC in a 2-4-4 configuration and 4-bit quantizers. Future work consists in taking into account non idealities in order to complete and refine circuits specifications. The chip will be designed to illustrate by measurements the subband approach interest for the DPD loop.

\section{ACKNOWLEDGMENT}

This work is supported by the "Lidex-Nanodesign" project funded by the IDEX Paris-Saclay, ANR-11-IDEX-0003-02.

\section{REFERENCES}

[1] F.M. Ghannouchi and O. Hammi. Behavioral Modeling and Predistortion. IEEE Microwave, 10(7):52-64, Dec. 2009.

[2] Ying Liu, Wensheng Pan, Shihai Shao, and Youxi Tang. A New Digital Predistortion for Wideband Power Amplifiers With Constrained Feedback Bandwidth. IEEE Microwave Wireless Compon. Lett., 23(12):683685, December 2013.

[3] Yuelin Ma, Yasushi Yamao, Yoshihiko Akaiwa, and Koji Ishibashi. Wideband Digital Predistortion Using Spectral Extrapolation of Bandlimited Feedback Signal. IEEE Trans. Circuits Syst. I, pages 1-10, 2014.

[4] D.-K.G. Pham, P. Desgreys, P. Loumeau, and T. Ridgers. Multi-Stage Noise Band Cancellation Sigma Delta Modulator for Digitisation of Distorted Signals. Electronics Letters, 48(10):560-562, 2012.

[5] D.-K.G. Pham, P. Desgreys, P. Loumeau, T. Ridgers, and G. Monnerie. High-Level Design of General Multi-Stage Noise Band Cancellation $\Sigma \Delta$ ADC Optimized for Nonlinearly Distorted Signals. Analog Integrated Circuits and Signal Processing, 77(2), 2013.

[6] E.J. vanderZwan and E.C. Dijkmans. A 0.2-mW CMOS $\Sigma \Delta$ Modulator for Speech Coding With $80 \mathrm{~dB}$ Dynamic Range. IEEE J. Solid-State Circuits, 31(12):1873-1880, Dec 1996.

[7] M. Keller, A. Buhmann, J. Sauerbrey, M. Ortmanns, and Y. Manoli. A Comparative Study on Excess-Loop-Delay Compensation Techniques for Continuous-Time $\Sigma \Delta$ Modulators. IEEE Transactions on Circuits and Systems I: Regular Papers, 55(11):3480-3487, Dec 2008.

[8] Lei Ding, G.T. Zhou, D.R. Morgan, Zhengxiang Ma, J.S. Kenney, Jaehyeong Kim, and C.R. Giardina. A Robust Digital Baseband Predistorter Constructed Using Memory Polynomials. IEEE Trans. Commun., 52(1):159-165, Jan 2004.

[9] M. Grimm, M. Allen, J. Marttila, M. Valkama, and R. Thoma. Joint Mitigation of Nonlinear RF and Baseband Distortions in Wideband Direct-Conversion Receivers. IEEE Trans. Microwave Theory Tech., 62(1):166-182, Jan 2014.

[10] 3GPP. TS 36.104 - E-UTRA BS Radio Transmission and Reception.

[11] R. Schreier . THE DELTA-SIGMA TOOLBOX v. 7.3, 2009. 